\title{
Século XXI: A filosofia de uma Odontologia minimamente invasiva, o que mudou do diagnóstico ao tratamento da cárie dental?
}

XXI Century: The philosophy of a mimimally invasive Dentistry, what has been changed did what's changed from the diagnosis to the treatment of dental caries?

Siglo XXI: La filosofía de una Odontología mínimamente invasiva, lo que cambió del diagnóstico al tratamiento de la caries dental?

Recebido: 08/09/2021 | Revisado: 17/09/2021 | Aceito: 24/09/2021 | Publicado: 26/09/2021

\author{
Dyele Kalynne Costa da Silva \\ ORCID: https://orcid.org/0000-0003-1861-8477 \\ Universidade Federal do Maranhão, Brasil \\ E-mail: dyele.kcs@discente.ufma.br \\ Carlos Felipe Sousa Menezes \\ ORCID: https://orcid.org/0000-0002-5938-1691 \\ Universidade Federal do Maranhão, Brasil \\ E-mail: carllos_fellipe@ @otmail.com \\ Anna Cecy Ribeiro Brito \\ ORCID: https://orcid.org/0000-0002-3703-7160 \\ Universidade Federal do Maranhão, Brasil \\ E-mail: anna.cecy@discente.ufma.br \\ Darlon Martins Lima \\ ORCID: https://orcid.org/0000-0001-9113-1206 \\ Universidade Federal do Maranhão, Brasil \\ E-mail: darlon.martins@ufma.br \\ Leily Macedo Firoozmand \\ ORCID: https://orcid.org/0000-0002-8634-188X \\ Universidade Federal do Maranhão, Brasil \\ E-mail: leily.firoozmand@ufma.br
}

\begin{abstract}
Resumo
Introdução: A cárie dentária é mundialmente a doença mais comum que influencia negativamente na qualidade de vida das pessoas. Com o avanço das pesquisas, o diagnóstico da lesão cariosa e uso de materiais e técnicas tem ganhado um caráter mais conservador. Objetivo: Avaliar por meio de evidências cientificas disponíveis na literatura, as atuais propostas de intervenções e protocolos para o tratamento da cárie, tendo como objetivo a máxima preservação e mínima intervenção na estrutura dental. Metodologia: Seleção dos artigos nas bases de dados, MEDLINE (Medical Literature Analysis and Retrieval Sistem on-line), PUBMED. A partir das palavras-chave "caries", "partial" e "removal”, 50 artigos (2010 a 2021) foram pré-selecionados. Seguindo os critérios de inclusão e exclusão, foram selecionados 17 artigos analisando de acordo com o manejo de lesões observando a severidade, o grau de atividade da cárie e tipo de material e técnica a serem empregados. Resultados: Os estudos demonstram uma maior compreensão da evolução da cárie, o estabelecimento de um diagnóstico mais criterioso e o desenvolvimento de materiais adesivos e bioativos tem possibilitado tratamentos minimamente invasivos. Diferenças significativas entre as técnicas de remoção total, parcial e em 2 passos (stepwise) de tecido cariado tem sido encontrada. sendo que a remoção total e em 2 passos aumentam as chances de injúria e/ou comunicação pulpar. Conclusão: A evolução dos materiais adesivos e uma maior compreensão do desenvolvimento da cárie possibilita o emprego de técnicas e materiais que garanta menor intervenção e maior sucesso clínico.
\end{abstract}

Palavras- chave: Cárie dental; Restauração dentária permanente; Testes de atividade de cárie dentária; Suscetibilidade à cárie dentária.

\begin{abstract}
Introduction: Dental caries is the most common disease worldwide and negatively influences people quality of life. With the advancement of research, the diagnosis of carious lesions and the use of materials and techniques has gained a more conservative character. Objective: To evaluate, through scientific evidence available in the literature, the current proposals for interventions and protocols for the treatment of caries, aiming at maximum preservation and minimum intervention in the dental structure. Methodology: Selection of articles in the databases, MEDLINE (Medical Literature Analysis and Retrieval Online System), PUBMED. From the keywords "caries", "partial" and "removal", 50 articles (2010 to 2021) were pre-selected. Following the inclusion and exclusion criteria, 17 articles were selected and analyzed
\end{abstract}


according to the management of injuries, observing the severity, the degree of activity of the caries and the type of material and technique to be used. Results: Studies demonstrated a greater understanding of the evolution of caries, the establishment of a more careful diagnosis and the development of adhesive and bioactive materials have enabled minimally invasive treatments. Significant differences between total, partial and 2-step (stepwise) removal of carious tissue have been found, being that the total removal and in 2 steps increase the chances of injury and/or pulp communication. Conclusion: The evolution of adhesive materials and a greater understanding of the development of caries enables the use of techniques and materials that guarantee less intervention and greater clinical success.

Keywords: Dental caries; Dental restoration permanent; Dental caries activity tests; Dental caries susceptibility.

\section{Resumen}

Introducción: La caries dental es la enfermedad más común a nivel mundial que influye negativamente en la calidad de vida de las personas. Con el avance de las investigaciones, el diagnóstico de lesiones cariosas y el uso de materiales y técnicas ha adquirido un carácter más conservador. Objetivo: Evaluar, a través de la evidencia científica disponible en la literatura, las propuestas actuales de intervenciones y protocolos para el tratamiento de la caries, con el objetivo de máxima preservación y mínima intervención en la estructura dentaria. Metodología: Selección de artículos en las bases de datos, MEDLINE (Sistema Online de Análisis y Recuperación de Literatura Médica), PUBMED. De las palabras clave "caries", "parcial" y "remoción", se preseleccionaron 50 artículos (2010 a 2021). Siguiendo los criterios de inclusión y exclusión, se seleccionaron y analizaron 17 artículos según el manejo de las lesiones, observando la gravedad, el grado de actividad de la caries y el tipo de material y técnica a utilizar. Resultados: Los estudios demuestran una mayor comprensión de la evolución de la caries, el establecimiento de un diagnóstico más cuidadoso y el desarrollo de materiales adhesivos y bioactivos han permitido tratamientos mínimamente invasivos. Se han encontrado diferencias significativas entre la remoción total, parcial y en 2 pasos (paso a paso) del tejido cariado. siendo que la remoción total y en 2 pasos aumentan las posibilidades de lesión y / o comunicación pulpar. Conclusión: La evolución de los materiales adhesivos y una mayor comprensión del desarrollo de la caries posibilita el uso de técnicas y materiales que garantizan una menor intervención y un mayor éxito clínico.

Palabras clave: Caries dentales; Restauración dental permanente; Pruebas de actividad de caries dental; Susceptibilidad a caries dentarias.

\section{Introdução}

A cárie dentária é mundialmente a doença mais comum (Hernández et al., 2014) e está associada a aspectos sociodemográficos e comportamentais dos pacientes (Veiga et al., 2015). Ocupa o primeiro lugar no ranking, quando estão envolvidos os dentes permanentes (2,3 bilhões de pessoas) de paciente adultos e o $12^{\circ}$ em dentes decíduos (560 milhões de crianças) de acordo com Global Burden of Disease Study de 2017 (GBD, 2018). Recentemente, a ORCA (Cariology Research Group of IADR), maior órgão de pesquisas em cariologia, trouxe terminologias atualizadas que consideram a cárie dentária como uma doença multifatorial, não transmissível, não infecciosa, crônica, induzida por biofilme, modulada por vários fatores biológicos, comportamentais, psicossociais e ambientais (Machiulskiene et al., 2020).

Estudos do impacto na qualidade de vida em pacientes jovens, tem observado benefícios do tratamento restaurador da cárie dentária e a necessidade do desenvolvimento de políticas públicas para este fim, já que em pacientes não tratados e/ou com falta de dentes, a qualidade de vida é afetada (Feldens et al., 2016).

Há alguns anos, grande parte das opções de tratamento para a cárie dental apresentava um caráter mais invasivo, visto que se acreditava que os microrganismos remanescentes na dentina afetada, se não removidos, permaneceriam por longo período de tempo (Sato \& Fusayama, 1976). Estudos em meados dos anos 2000 já relatam não haver necessidade de remover totalmente o esmalte afetado para a restauração devido ao seu grande potencial remineralizante, contudo, muitos dentistas continuavam a intervir nestas lesões (Bader et al., 2001; Rindal et al., 2012). Os avanços em estudos sobre a dentina cariada evidenciaram que a remoção "total" da dentina afetada pela cárie, além da dificuldade clínica, aumentava os riscos de exposição pulpar. Logo, as evidências cientificas tem apontado que só o fato da cavidade ser bem selada, não haveria condições significativas de agressão à polpa e o processo de carioso não prosseguiria (Kidd, 2000). Além disso, o desenvolvimento de técnicas como a stepwise (remoção da cárie em dois passos/estágios) indicavam que poderia haver um aumento na preservação pulpar em lesões cariosas profundas (Hayashi et al., 2011). 
A partir desta perspectiva, o tratamento das lesões cariosas está caminhando para uma filosofia de intervenção mínima (MI). Tem sido relatada a importância do tratamento individualizado de acordo com o risco de cárie, a detecção e o tratamento precoce de lesões não cavitadas, ou seja grande ênfase tem sido dada à realização de abordagens preventivas e restauradoras minimamente invasivas (Banerjee \& Doméjean, 2013; Frencken et al., 2012; Lee et al., 2018; Ngo \& Opsahl-Vital, 2014; Tyas et al., 2000). A Caries Care International elaborou um plano estruturado que pode levar a intervenções de forma personalizadas. O denominado "4D", foi elaborado com base em quatro etapas: DETERMINAR (determine) o risco de cárie; DETECTAR (detect) as lesões, escalonando sua gravidade e atividade; DECIDIR (decide) sobre o planejamento mais adequado; e, FAZER (do) os cuidados preventivos em lesões iniciais não cavitadas e tratamento restaurador conservador de lesões cavitadas (Martignon et al., 2021).

Dentro deste contexto, o manejo da cárie considerando a atividade da lesão, a presença de cavitação e a profundidade da lesão inclui intervenções não invasivas, microinvasivas e minimamente invasivas (Slayton et al., 2018). Assim, embasados na ausência e necessidade de protocolos bem definidos de acordo com os conceitos atuais, que associam as características da lesão cariosa alinhados às técnicas e/ou materiais indicados no procedimento restaurador, este estudo tem como objetivo avaliar as evidências cientificas disponíveis na literatura nos últimos 10 anos sobre as intervenções e protocolos em lesões de cárie eficazes e minimamente invasivos. Assim, será possível estabelecer manobras que permitam manter o máximo de estrutura dental com sucesso clínico, por longos períodos de tempo.

\section{Metodologia}

Para a elaboração desta revisão integrativa as seguintes etapas foram percorridas: identificação do tema e seleção da hipótese ou questão de pesquisa; estabelecimento de critérios para inclusão e exclusão de estudos/amostragem ou busca na literatura; definição das informações a serem extraídas dos estudos selecionados/ categorizados; avaliação dos estudos incluídos; interpretação dos resultados; e apresentação da revisão/síntese do conhecimento (Mendes et al., 2008).

\section{- Questão de Pesquisa}

O estudo da condição do tema gerou a seguinte questão de pesquisa: Quais os protocolos minimamente invasivos no manejo de lesões cariosas de acordo com os critérios pré-estabelecidos de severidade e atividade da lesão?

\section{- Critérios de Inclusão/Exclusão}

Os critérios de inclusão dos artigos definidos, inicialmente, para a presente revisão integrativa foram: artigos publicados em inglês, com os resumos disponíveis nas Bases de Dados selecionadas, no período compreendido entre 2010-2021. As palavras-chave utilizadas foram "caries", "partial, e "removal" e a busca foi realizada pelo acesso on-line. Foram excluídos artigos duplicados e que se tratavam de técnicas invasivas com injúria pulpar ou sobre as condições pulpares após comunicação com a cavidade pulpar.

\section{- Coleta de Dados}

Para a seleção dos artigos foram utilizadas duas bases de dados internacionais, a saber: o MEDLINE (Medical Literature Analysis and Retrieval Sistem on-line), o PUBMED. Dessa forma, procurou-se ampliar o âmbito da pesquisa, minimizando possíveis vieses nessa etapa do processo de elaboração da revisão integrativa.

\section{- Análise e Seleção dos Estudos}

Para a análise e posterior síntese dos artigos que atenderam aos critérios de inclusão foi organizado um quadro sinóptico especialmente construído para esse fim. Foram contemplados os seguintes aspectos: autor, título, tipo de estudo e intervenção 
resultados. Assim, artigos publicados cuja metodologia adotada permitissem obter evidências fortes níveis 1, 2 e 3 (Quadro1) foram incluídos no estudo.

Quadro 1 - Quadro resumo do nível de relevância dos estudos a partir da caracterização do estudo.

\begin{tabular}{|l|l|}
\hline NÍVEL & DESCRIÇÃO \\
\hline 1 & $\begin{array}{l}\text { Evidências resultantes de meta-análise de múltiplos ensaios clínicos controlados } \\
\text { randomizados }\end{array}$ \\
\hline 2 & Evidências de estudos individuais com design experimental \\
\hline 3 & Evidências de estudos experimentais \\
\hline 4 & Evidência de estudos descritivos (não experimental) ou com abordagem qualitativa \\
\hline 5 & Evidências de relatos de caso ou de experiência \\
\hline 6 & Evidências baseadas em opiniões de especialistas \\
\hline
\end{tabular}

Souza et al., (2010).

Para esquematizar o processo de seleção dos artigos, foi necessário analisar os artigos que atendiam as respostas da questão norteadora. Para que houvesse a seleção dos artigos, guiados pela leitura título e depois pela leitura do resumo, foram eliminados ou escolhidos à medida que respondiam a temática em estudo. De forma sucinta, foram lidos 24 artigos, porém após a análise do quadro sinóptico, apenas 17 foram escolhidos por obedecerem aos critérios já mencionados acima (Figura 1).

Figura 1: Fluxograma da seleção de artigos.

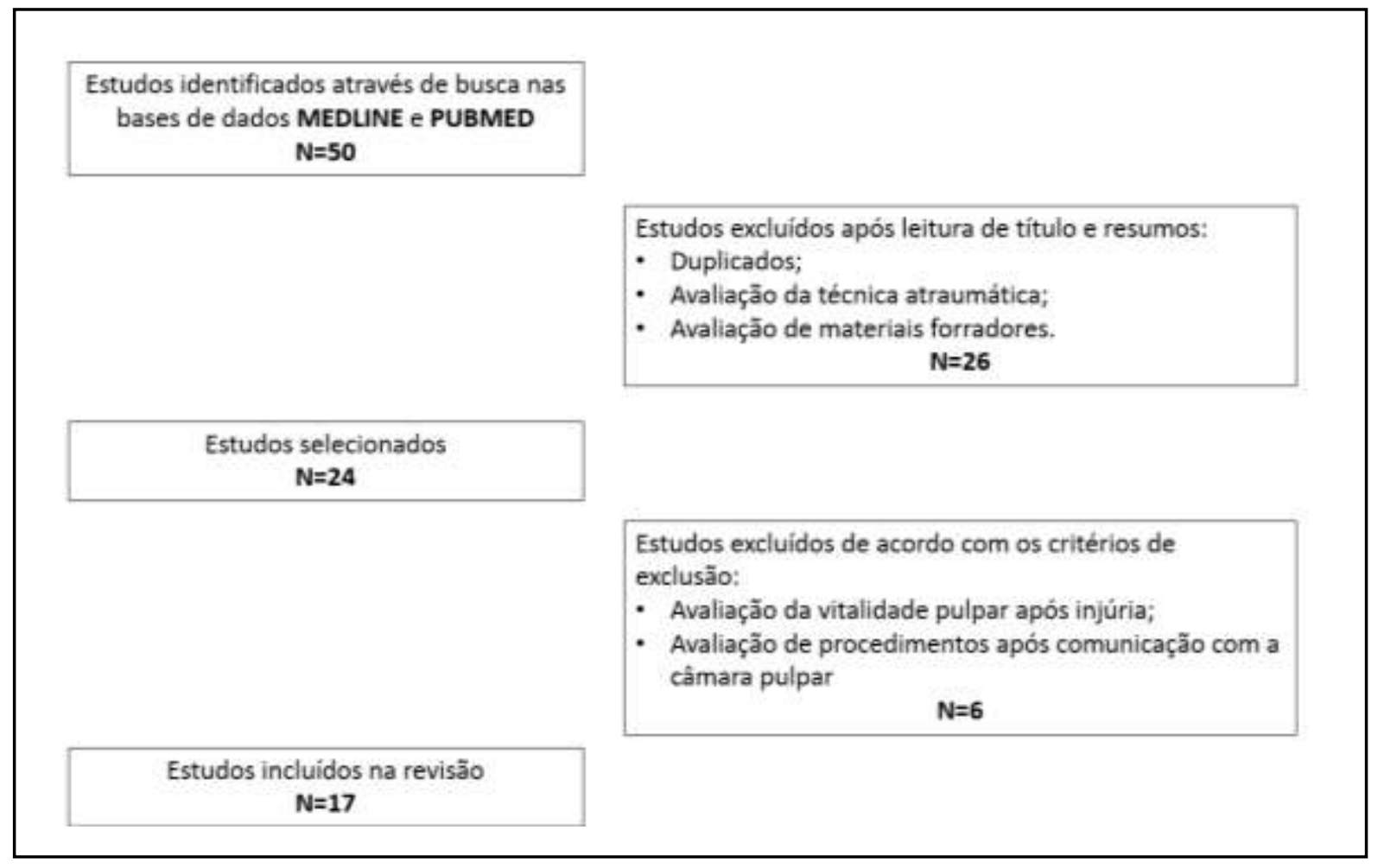

Fonte: Autores. 


\section{Resultados e Discussão}

O avanço em estudos de materiais como adesivos e com partículas bioativas que promovem a evolução de técnicas minimamente invasivas, realçam a necessidade de estudos de protocolos que visam associar a doença (diagnóstico) com protocolos de atendimentos bem sedimentados. Assim, a partir da seleção de artigos obtidos após busca em base de dados, e, exclusão de acordo com os critérios estabelecidos e nível de relevância dos estudos (Quadro 1), foi realizada a leitura sucinta dos artigos selecionados. A partir da avaliação destes estudos, obteve-se o quadro sinóptico indicando os principais aspectos de relevância para esta análise (Quadro 2).

Diante da compreensão da importância da realização de uma Odontologia Minimamente Invasiva, foram observados os critérios estabelecidos pelos "4D" quatro passos (DETERMINAR o risco de cárie; DETECTAR a gravidade e atividade; DECIDIR o tratamento mais adequado; e, REALIZAR o tratamento (Martignon et al., 2021) para a avaliação e análise dos estudos publicados. Observou-se que nenhum dos estudos selecionados, no presente estudo, evidenciou os critérios de classificação da severidade ou atividade das lesões cariosas para a avaliação dos tratamentos. Além disso, as considerações quanto à estrutura remanescente e a seleção do material e ou técnica restauradora não foram bem detalhadas nos estudos avaliados.

Os aspectos referentes à remoção do tecido cariado têm sido ressaltados em alguns estudos, que demonstram haver diferenças significativas entre as técnicas de remoção total, parcial e stepwise (Quadro 2). Tanto a remoção total quanto a remoção em dois passos clínicos do tecido cariado aumentam as chances de injúria e/ou comunicação pulpar (Maltz et al., 2012; Manton, 2013; Mattos et al., 2014; Hoefler et al., 2016; Villat et al., 2016; Zhang , 2017; Bjørndal et al., 2017; Maltz et al., 2018; Khokhar e Tewari, 2018; Giacaman et al., 2018; Singh et al., 2019; Ricketts et al., 2019; Pozos-guillén et al., 2021) Além disso, estudos também evidenciam que não há diferença estatisticamente diferentes quando do uso de materiais forradores ou inertes no selamento de cavidades profundas (Corralo e Maltz, 2013; Giacaman et al., 2018).

Alguns estudos (Giacaman et al., 2018; Pozos-Guillén et al., 2021; Ricketts et al., 2019), associam um protocolo de manejo das lesões, fornecendo opção de materiais que podem ser utilizados no procedimento restaurado, contudo, não associam estas informações ao grau de severidade e atividade da lesão (ICDAS II e Nyvad).

A remoção parcial de cárie tem apresentado resultados positivos independentemente do material restaurador final selecionado (Ricketts et al., 2019) para restauração do dente. Esta conduta, associada à avaliação da resposta pulpar e aos índices de gravidade e atividade de cárie, auxiliam na seleção da melhor técnica a ser aplicada no tratamento da lesão cariosa. Porém, ainda há a necessidade de mais estudos clínicos randomizados que avaliem técnicas que propiciem menor risco de injúria pulpar e menor desconforto ao paciente em relação às técnicas mais invasivas (Banerjee \& Doméjean, 2013; Hoefler et al., 2016; Manton, 2013; Mattos et al., 2014).

A partir da análise e avaliação dos estudos incluídos nesta bibliografia revisada foi possível formular um protocolo para avaliação do risco, grau de severidade e atividade da lesão de cárie associando estes parâmetros aos tratamentos e materiais indicados. O Quadro 3 traz um esquema que leva em consideração esses critérios e auxilia na tomada de decisão do diagnóstico ao plano de tratamento de lesões cariosas em dentina. 
Research, Society and Development, v. 10, n. 12, e440101220385, 2021

(CC BY 4.0) | ISSN 2525-3409 | DOI: http://dx.doi.org/10.33448/rsd-v10i12.20385

Quadro 2: Quadro Sinóptico dos estudos que abordam a remoção parcial do tecido cariado.

\begin{tabular}{|c|c|c|c|}
\hline AUTOR & TÍTULO & TIPO DE ESTUDO & INTERVENÇÃO/ RESULTADOS \\
\hline $\begin{array}{l}\text { MALTZ et al., } \\
2012\end{array}$ & $\begin{array}{l}\text { Randomized trial of partial } \\
\text { vs. stepwise caries removal: } \\
\text { 3-year follow-up }\end{array}$ & Estudo clínico randomizado & $\begin{array}{l}\text { Avaliação da eficácia - remoção parcial de cárie e escavação em etapas ( stepwise) - com acompanhamento } \\
\text { de } 3 \text { anos. Os resultados sugerem que não há necessidade de reabrir uma cavidade e realizar uma segunda } \\
\text { escavação para que a vitalidade pulpar seja preservada }\end{array}$ \\
\hline $\begin{array}{l}\text { BANERJEE, } \\
2012\end{array}$ & $\begin{array}{l}\text { "MI" caries management--an } \\
\text { overview }\end{array}$ & Revisão de literatura & $\begin{array}{l}\text { Revisão de literatura sobre Odontologia Minimamente Invasiva - estratégias minimamente invasivas de } \\
\text { remoção de cárie e materiais usados. Instrumentos de escavação, incluindo brocas / peças de mão, } \\
\text { escavadeiras manuais, agentes quimio-mecânicos e / ou abrasivos a ar que limitam a remoção de cárie } \\
\text { seletivamente para a dentina infectada por cárie mais superficial e a remoção parcial da dentina afetada por } \\
\text { cárie quando necessário, ajudam a criar cavidades menores com margens de esmalte / dentina saudáveis. }\end{array}$ \\
\hline MANTON, 2013 & $\begin{array}{l}\text { Partial caries removal may } \\
\text { have advantages but limited } \\
\text { evidence on restoration } \\
\text { survival }\end{array}$ & Revisão de literatura & $\begin{array}{l}\text { Comparar a remoção gradual, parcial e sem cárie dentinária com a remoção completa de cárie em dentes } \\
\text { decíduos e permanentes. Para o tratamento da cárie dentinária, tanto a escavação gradativa quanto a parcial } \\
\text { mostraram vantagem clínica sobre a remoção completa da cárie, reduzindo a incidência de exposição pulpar } \\
\text { em dentes primários, vitais e cariados sem sintomas, bem como em dentes permanentes. A revisão não } \\
\text { encontrou nenhuma diferença nos sinais ou sintomas de doença pulpar entre a escavação gradual e a } \\
\text { remoção completa da cárie. }\end{array}$ \\
\hline $\begin{array}{l}\text { CORRALO; } \\
\text { MALTZ, } 2013\end{array}$ & $\begin{array}{l}\text { Clinical and ultrastructural } \\
\text { effects of different } \\
\text { liners/restorative materials on } \\
\text { deep carious dentin: a } \\
\text { randomized clinical trial }\end{array}$ & Estudo clínico randomizado & $\begin{array}{l}\text { Avaliação do efeito do cimento de hidróxido de cálcio (CHC) e cimento de ionômero de vidro (GIC) na } \\
\text { dentina cariada e infecções bacterianas após a remoção parcial de cárie e selamento. Os achados indicaram } \\
\text { que a remoção das lâminas superficiais da dentina necrótica e desmineralizada e o selamento da cavidade } \\
\text { promovem a contenção da cárie dentinária, independentemente do uso de forrador. Os resultados sugerem } \\
\text { que o efeito do uso de CHC ou GIC na retenção de cárie dentinária não é superior ao uso de um material } \\
\text { inerte. }\end{array}$ \\
\hline $\begin{array}{l}\text { MALTZ et al., } \\
2013\end{array}$ & $\begin{array}{l}\text { Partial removal of carious } \\
\text { dentine: a multicenter } \\
\text { randomized controlled trial } \\
\text { and } 18 \text {-month follow-up } \\
\text { results }\end{array}$ & Estudo clínico randomizado & $\begin{array}{l}\text { Avaliação da eficácia da remoção parcial de dentina cariosa e restauração em sessão única (Remoção parcial } \\
\text { da cárie) e escavação gradual (stepwise). A retenção de dentina cariada não interfere na vitalidade pulpar. Os } \\
\text { dados deste estudo de } 18 \text { meses sugerem que o procedimento de reabertura da cavidade para remover a } \\
\text { dentina infectada residual não é necessário. }\end{array}$ \\
\hline $\begin{array}{l}\text { HERNÁNDEZ; } \\
\text { COBB; JR, } 2014\end{array}$ & $\begin{array}{l}\text { Current strategies in dentin } \\
\text { remineralization }\end{array}$ & $\begin{array}{l}\text { Ensaio clínico prospectivo } \\
\text { randomizado controlado }\end{array}$ & $\begin{array}{l}\text { Avaliar clinicamente e, qualitativamente a dentina residual afetada. Os achados sugerem que há uma } \\
\text { estabilização da lesão e aparente remineralização após } 3 \text { meses. }\end{array}$ \\
\hline
\end{tabular}




\begin{tabular}{|c|c|c|c|}
\hline $\begin{array}{l}\text { MATTOS; } \\
\text { SOARES; } \\
\text { RIBEIRO, } 2014\end{array}$ & $\begin{array}{l}\text { Current status of conservative } \\
\text { treatment of deep carious } \\
\text { lesions. }\end{array}$ & Revisão de literatura & $\begin{array}{l}\text { O objetivo deste artigo foi descrever e discutir as principais técnicas de remoção de tecido cariado, de acordo } \\
\text { com evidências científicas. Foi observado que tanto a escavação gradativa quanto a cárie parcial a remoção } \\
\text { do tecido apresentou menores taxas de exposição pulpar e maiores taxas de sucesso. }\end{array}$ \\
\hline $\begin{array}{l}\text { HOEFLER; } \\
\text { NAGAOKA; } \\
\text { MILLER, } 2016\end{array}$ & $\begin{array}{l}\text { Long-term survival and } \\
\text { vitality outcomes of } \\
\text { permanent teeth following } \\
\text { deep caries treatment with } \\
\text { step-wise and partial-caries- } \\
\text { removal: A Systematic } \\
\text { Review. }\end{array}$ & Revisão sistemática & $\begin{array}{l}\text { Uma revisão sistemática foi realizada para comparar a sobrevivência a longo prazo de dentes permanentes } \\
\text { afetados por cárie dentinária profunda tratados com remoção parcial de cárie versus dentes semelhantes } \\
\text { tratados com técnicas de remoção passo a passo de cárie (stepwise). Qualquer reclamação de uma vantagem } \\
\text { terapêutica ao tratar dentes permanentes com cárie dentinária profunda usando remoção parcial em vez de } \\
\text { stepwise é apoiado por um baixo número de estudos que consistem em evidências orientadas para o paciente } \\
\text { de qualidade limitada. }\end{array}$ \\
\hline $\begin{array}{l}\text { VILLAT et al., } \\
2016\end{array}$ & $\begin{array}{l}\text { One-step partial or complete } \\
\text { caries removal and bonding } \\
\text { with antibacterial or } \\
\text { traditional self-etch } \\
\text { adhesives: study protocol for } \\
\text { a randomized controlled trial. }\end{array}$ & Estudo clínico randomizado & $\begin{array}{l}\text { Os objetivos deste estudo foram comparar a eficácia da remoção da dentina cariada parcial versus total em } \\
\text { lesões profundas (objetivo primário) e o uso de um antibacteriano versus um autocondicionante tradicional } \\
\text { de duas etapas sistema adesivo (objetivo secundário principal). }\end{array}$ \\
\hline $\begin{array}{l}\text { ZHANG; OU; } \\
\text { ZHANG, } 2017\end{array}$ & $\begin{array}{l}\text { Curative effects of partial } \\
\text { caries removal in permanent } \\
\text { molars with deep dental } \\
\text { caries. }\end{array}$ & Estudo Clínico & $\begin{array}{l}\text { Avaliar a eficácia clínica da remoção incompleta de cárie em molares permanentes com cárie dentária } \\
\text { profunda. A remoção parcial da cárie pode reduzir o risco de exposição pulpar em cáries profundas de } \\
\text { molares permanentes e o desconforto durante o tratamento. O efeito clínico é bom, o que merece ampla } \\
\text { aplicação clínica. }\end{array}$ \\
\hline $\begin{array}{l}\text { BJØRNDAL et } \\
\text { al., } 2017\end{array}$ & $\begin{array}{l}\text { Randomized Clinical Trials } \\
\text { on Deep Carious Lesions: } 5 \text { - } \\
\text { Year Follow-up. }\end{array}$ & Ensaio clínico randomizado & $\begin{array}{l}\text { O objetivo deste artigo era relatar o resultado de } 5 \text { anos com lesões de cárie bem definidas radiograficamente } \\
\text { que se estendem para a polpa. Procedimentos de capeamento de polpa pode ser um tratamento duvidoso em } \\
\text { adultos com cárie profunda. Ensaios clínicos randomizados são necessários para expandir as evidências de se } \\
\text { uma remoção seletiva de cárie deve ser preferida para uma remoção gradual em } 2 \text { estágios de tecido cariado } \\
\text { profundo. }\end{array}$ \\
\hline $\begin{array}{l}\text { MALTZ et al., } \\
2018\end{array}$ & $\begin{array}{l}\text { Partial caries removal in } \\
\text { deep caries lesions: a 5-year } \\
\text { multicenter randomized } \\
\text { controlled trial. }\end{array}$ & Estudo clínico randomizado & $\begin{array}{l}\text { Comparar os resultados da escavação em etapas (stepwise) e remoção parcial de cárie (RPC) em relação à } \\
\text { manutenção da vitalidade pulpar em lesões de cárie profundas ao longo de } 5 \text { anos. Em conclusão, a RPC } \\
\text { mostrou um maior sucesso quando comparada a stepwise com mais de } 5 \text { anos de monitoramento. Alguns } \\
\text { pacientes não retornarem para completar o stepwise o que impactou negativamente no desempenho clínico } \\
\text { desta técnica. }\end{array}$ \\
\hline
\end{tabular}


Research, Society and Development, v. 10, n. 12, e440101220385, 2021

(CC BY 4.0) | ISSN 2525-3409 | DOI: http://dx.doi.org/10.33448/rsd-v10i12.20385

\begin{tabular}{|c|c|c|c|}
\hline $\begin{array}{l}\text { KHOKHAR; } \\
\text { TEWARI, } 2018\end{array}$ & $\begin{array}{l}\text { Outcomes of Partial and } \\
\text { Complete Caries Excavation } \\
\text { in Permanent Teeth: A } 18 \\
\text { Month Clinical Study }\end{array}$ & Estudo clínico randomizado & $\begin{array}{l}\text { Avaliar molares permanentes maduros com lesões cariosas profundas tratados com remoção parcial de cárie } \\
\text { e remoção total de cárie. A remoção parcial de tecido cariado pode ser uma opção de tratamento eletiva para } \\
\text { os dentes permanentes maduros com lesões cariosas profundas. }\end{array}$ \\
\hline $\begin{array}{l}\text { GIACAMAN et } \\
\text { al., } 2018\end{array}$ & $\begin{array}{l}\text { Evidence-based strategies for } \\
\text { the minimally invasive } \\
\text { treatment of carious lesions: } \\
\text { Review of the literature. }\end{array}$ & Revisão de Literatura & $\begin{array}{l}\text { Avaliar várias alternativas de mínima intervenção para o tratamento da lesão de cárie, incluindo o uso de } \\
\text { selantes, infiltração, tratamento restaurador atraumático (ART) e remoção seletiva de tecido cariado (por } \\
\text { exemplo, capeamento pulpar indireto, remoção gradual ou remoção seletiva para dentina mole). A } \\
\text { preservação da estrutura dentária através do uso de tratamento minimamente invasivo para lesões não } \\
\text { cavitadas e cavitadas é apoiada por evidências moderadas. }\end{array}$ \\
\hline $\begin{array}{l}\text { SINGH; } \\
\text { MITTAL; } \\
\text { TEWARI, } 2019\end{array}$ & $\begin{array}{l}\text { Effect of Different Liners on } \\
\text { Pulpal Outcome after Partial } \\
\text { Caries Removal: A } \\
\text { Preliminary } 12 \text { Months } \\
\text { Randomised Controlled Trial }\end{array}$ & $\begin{array}{l}\text { Estudo clínico randomizado duplo } \\
\text { cego }\end{array}$ & $\begin{array}{l}\text { Avaliar molares com cárie profundas envolvendo dois terços ou mais da dentina, tratados com hidróxido de } \\
\text { cálcio, ionômero de vidro modificado por resina e grupos sem forrador após Remoção parcial de cárie. A } \\
\text { remoção parcial de cárie tem uma grande taxa de sucesso ao tratar lesões cariosas profundas em dentes } \\
\text { permanentes após } 12 \text { meses de acompanhamento, indicando que a permanência de uma dentina afetada não } \\
\text { interfere na vitalidade pulpar ou na restauração superficial. Além disso, o sucesso do tratamento é } \\
\text { independente do material utilizado sobre a dentina desmineralizada. }\end{array}$ \\
\hline $\begin{array}{l}\text { RICKETTS et al., } \\
2019\end{array}$ & $\begin{array}{l}\text { Operative caries management } \\
\text { in adults and children }\end{array}$ & Revisão de literatura & $\begin{array}{l}\text { Através de uma revisão de literatura avaliar os efeitos da remoção gradual, parcial ou nenhuma de cárie } \\
\text { dentinária em comparação com a remoção completa de cárie para o tratamento de cárie dentária em dentes } \\
\text { decíduos e permanentes previamente não restaurados. A escavação gradual e parcial reduziu a incidência de } \\
\text { exposição pulpar em dentes primários, vitais, cariados e sem sintomas, assim como em dentes permanentes. } \\
\text { Portanto, essas técnicas apresentam vantagem clínica sobre a remoção completa de cárie no tratamento da } \\
\text { cárie dentinária. }\end{array}$ \\
\hline $\begin{array}{l}\text { POZOS- } \\
\text { GUILLÉN et al., } \\
2021\end{array}$ & $\begin{array}{l}\text { Management of dental caries } \\
\text { lesions in Latin American and } \\
\text { Caribbean countries }\end{array}$ & Revisão de literatura & $\begin{array}{l}\text { Busca de evidências em ensaios clínicos sobre as atuais estratégias para o manejo das lesões de cárie na } \\
\text { dentição decídua e permanente com o objetivo de orientar os dentistas da América Latina e Caribe. Remoção } \\
\text { seletiva de tecidos de cariado até a dentina amolecida sobre o teto da câmara pulpar seguido por restauração } \\
\text { definitiva em uma sessão deve ser o tratamento recomendado de escolha para lesões profundas de cárie } \\
\text { assintomáticas; }\end{array}$ \\
\hline
\end{tabular}

Fonte: Autores. 
Quadro 3 - Quadro esquemático com a caracterização da cavidade de acordo com o grau de severidade da lesão (ICDAS II), o grau de atividade da cárie (Nyvad), a vitalidade pulpar e o material a e técnica a serem trabalhadas de acordo com a caracterização da lesão.

\begin{tabular}{|c|c|c|c|c|}
\hline \multicolumn{2}{|l|}{$\begin{array}{l}\text { DESENHO ESQUEMÁTICO } \\
\text { TIPO DE CAVIDADE }\end{array}$} & $\begin{array}{c}\text { ICDAS } \\
\text { e } \\
\text { ACOMETIMENTO }\end{array}$ & $\begin{array}{l}\text { ATIVIDADE DE } \\
\text { CÁRIE }\end{array}$ & TRATAMENTO \\
\hline & & (0) - Hígido & - & - \\
\hline & A & $\begin{array}{l}\text { (1) - Fossa e } \\
\text { fissuras }\end{array}$ & SIM ou NÃO & $\begin{array}{l}\text { Controle de higiene/ Selante/ } \\
\text { Infiltrado resinoso }\end{array}$ \\
\hline & B & $\begin{array}{l}\text { (2) - Um pouco } \\
\text { além das fossas } \\
\text { e fissuras }\end{array}$ & SIM ou NÃO & $\begin{array}{l}\text { Controle de higiene/ } \\
\text { Produtos fluoretados/ } \\
\text { Selante/ Infiltrado resinoso }\end{array}$ \\
\hline & $\mathrm{C}$ & $\begin{array}{l}\text { (3) - Além das } \\
\text { fossas e } \\
\text { fissuras, antes } \\
\text { de Dentina }\end{array}$ & SIM ou NÃO & Selante/ Infiltrado resinoso \\
\hline & D & $\begin{array}{l}\text { (4) - "Sombra" em } \\
\text { dentina }\end{array}$ & SIM ou NÃO & $\begin{array}{l}\text { Selante/ Infiltrado } \\
\text { resinoso/Restauração com } \\
\text { Resina Composta }\end{array}$ \\
\hline & $\mathbf{E}$ & $\begin{array}{l}\text { (5) - Base em } \\
\text { dentina }\end{array}$ & SIM & $\begin{array}{l}\text { Stepwise + Material } \\
\text { Inomérico/Bioativo }\end{array}$ \\
\hline & & & NÃO & $\begin{array}{l}\text { Remoção parcial de cárie + } \\
\text { Material Inomérico/Bioativo + } \\
\text { Resina Composta }\end{array}$ \\
\hline & $\mathbf{F}$ & $\begin{array}{l}\text { (6) - Cavidade em } \\
\text { dentina }\end{array}$ & $\begin{array}{l}\text { SIM (Teste } \\
\text { térmico } \\
\text { positivo) }\end{array}$ & $\begin{array}{l}\text { Stepwise + Material } \\
\text { Inomérico/Bioativo }\end{array}$ \\
\hline & & & $\begin{array}{l}\text { NÃO (Teste } \\
\text { térmico } \\
\text { positivo) }\end{array}$ & $\begin{array}{l}\text { Remoção parcial de cárie + } \\
\text { Material Inomérico/Bioativo + } \\
\text { Resina Composta }\end{array}$ \\
\hline
\end{tabular}

Fonte: Autores.

A abordagem no tratamento da cárie, há alguns anos atrás, era a remoção invasiva, onde após a remoção total do tecido cariado, o mesmo era substituído por um material de restaurador. Diante da grande prevalência da cárie, da compreensão do processo carioso e das limitações dos materiais disponíveis, tornavam a remoção total da cárie a melhor opção terapêutica (Murdoch-Kinch \& McLean, 2003). A evolução dos materiais adesivos e da compreensão do processo de desenvolvimento da cárie possibilitou o desenvolvimento da Odontologia Minimamente Invasiva (Tyas et al., 2000).

No auxílio ao diagnóstico visual para determinar a cárie e seu grau de comprometimento da estrutura dental, a literatura aponta o ICDAS (International Caries Detection and Assessment System) (Quadro 3) como o critério de avaliação mais reconhecido e utilizado (Giacaman et al., 2018). Ele possibilita a caracterização da severidade da lesão cariosa, desde as menores variações nos sinais visuais (Ismail et al., 2007). É importante salientar que além da extensão da doença, o status de atividade também deve ser considerado, visto que lesões com mesmo código ICDAS, podem ter status de atividade diferente. Assim, o critério Nyvad considera tanto severidade como a atividade da cárie (Nyvad et al., 1999). Além disso, um aspecto importante é a avaliação da vitalidade pulpar e, os estudos, em sua grande maioria, consideram critérios em comum de diagnóstico (Tedesco et al., 2020), como teste térmico e avaliação clínica e radiográfica (Dimitraki et al., 2019). 
A partir desta compreensão, fica mais seguro determinar, detectar, diagnosticar e decidir a melhor opção de tratamento de forma individualizada. Nas lesões de ICDAS 1 a 3, já é bem sedimentado o tratamento minimamente invasivo, visto que são lesões não cavitadas e/ou limitadas ao esmalte que vão desde a instrução de higiene à aplicação de materiais fluoretados e uso de selantes e infiltrado resinoso (Coelho et al., 2019; Elhennawy et al., 2019; Lygidakis, 2010). Somado a isso, entende-se que as alternativas e/ou necessidade de forramento/capeamento com cimentos como o cimento de hidróxido de cálcio, que merecem maiores investigações a respeito da eficácia de seu uso, por haver indícios de que a médio e longo prazo tem resultados semelhantes ao uso de materiais inertes (Cushley et al., 2021; Rosa et al., 2019).

A melhoria nas propriedades físicas e adesivas dos materiais, possibilitaram a utilização de resinas compostas diretas em diferentes casos, inclusive nos mais extensos com ampla perda de estrutura dental. A técnica direta, além apresentam longevidade aceitável, tem baixos custos biológico e financeiro (Loomans et al., 2018; Mesko et al., 2016).

Por fim, os trabalhos que visam cavidades profundas de cárie se atentam mais ao comprometimento e/ou à resposta pulpar à curto e médio prazo (Bjørndal et al., 2017; Maltz et al., 2018; Zhang et al., 2017), à remoção total ou parcial da cárie (Pozos-Guillén et al., 2021; Ricketts et al., 2019), sem ao menos, por vezes, levar em consideração fatores como função, estética e durabilidade dos procedimentos e, fundamental, a atividade e severidade da cárie como critério na tomada de decisão quanto ao manejo dessas lesões.

\section{Conclusão}

A avaliação da lesão de cárie é de fundamental importância para seleção de protocolo de atendimento individualizado e que permita o uso de técnicas minimamente invasivas e conservadoras da estrutura e da vitalidade dental. Com a compreensão dos índices de avaliação de cárie como extensão da lesão e atividade de cárie e a evolução dos materiais adesivos, sugere-se um protocolo de manejo de lesões com comprometimento dentinário que leva em consideração a resposta pulpar, os índices de avaliação de severidade e atividade de cárie e o material restaurador indicado.

\section{Referências}

Bader, J. D., Shugars, D. A., \& Bonito, A. J. (2001). A systematic review of selected caries prevention and management methods. Community Dentistry and Oral Epidemiology, 29(6), 399-411. https://doi.org/10.1034/J.1600-0528.2001.290601.X

Banerjee, A. (2012). “MI” caries management--an overview. Annals of the Royal Australasian College of Dental Surgeons, $21,43-48$.

Banerjee, A., \& Doméjean, S. (2013). The contemporary approach to tooth preservation: minimum intervention (MI) caries management in general practice. Primary Dental Journal, 2(3), 30-37. https://doi.org/10.1308/205016813807440119

Bjørndal, L., Fransson, H., Bruun, G., Markvart, M., Kjældgaard, M., Näsman, P., Hedenbjörk-Lager, A., Dige, I., \& Thordrup, M. (2017). Randomized Clinical Trials on Deep Carious Lesions: 5-Year Follow-up. Journal of Dental Research, 96(7), 747-753. https://doi.org/10.1177/0022034517702620

Coelho, A. S. E. da C., Mata, P. C. M., Lino, C. A., Macho, V. M. P., Areias, C. M. F. G. P., Norton, A. P. M. A. P., \& Augusto, A. P. C. M. (2019). Dental hypomineralization treatment: A systematic review. Journal of Esthetic and Restorative Dentistry: Official Publication of the American Academy of Esthetic Dentistry ... [et Al.], 31(1), 26-39. https://doi.org/10.1111/JERD.12420

Corralo, D. J., \& Maltz, M. (2013). Clinical and ultrastructural effects of different liners/restorative materials on deep carious dentin: a randomized clinical trial. Caries Research, 47(3), 243-250. https://doi.org/10.1159/000345648

Cushley, S., Duncan, H. F., Lappin, M. J., Chua, P., Elamin, A. D., Clarke, M., \& El-Karim, I. A. (2021). Efficacy of direct pulp capping for management of cariously exposed pulps in permanent teeth: a systematic review and meta-analysis. International Endodontic Journal, 54(4), 556-571. https://doi.org/10.1111/IEJ.13449

Dimitraki, D., Papageorgiou, S. N., \& Kotsanos, N. (2019). Direct pulp capping versus pulpotomy with MTA for carious primary molars: a randomised clinical trial. European Archives of Paediatric Dentistry: Official Journal of the European Academy of Paediatric Dentistry, 20(5), 431-440. https://doi.org/10.1007/S40368-019-00419-7

Elhennawy, K., Krois, J., Jost-Brinkmann, P. G., \& Schwendicke, F. (2019). Outcome and comparator choice in molar incisor hypomineralisation (MIH) intervention studies: A systematic review and social network analysis. BMJ Open, 9(8). https://doi.org/10.1136/BMJOPEN-2018-028352

Feldens, C. A., Ardenghi, T. M., Dullius, A. I. D. S., Vargas-Ferreira, F., Hernandez, P. A. G., \& Kramer, P. F. (2016). Clarifying the Impact of Untreated and Treated Dental Caries on Oral Health-Related Quality of Life among Adolescents. Caries Research, 50(4), 414-421. https://doi.org/10.1159/000447095 
Frencken, J. E., Peters, M. C., Manton, D. J., Leal, S. C., Gordan, V. V., \& Eden, E. (2012). Minimal intervention dentistry for managing dental caries - a review: report of a FDI task group. International Dental Journal, 62(5), 223-243. https://doi.org/10.1111/IDJ.12007

GBD, G. B. of D. (2018). Global, regional, and national incidence, prevalence, and years lived with disability for 354 diseases and injuries for 195 countries and territories, 1990-2017: a systematic analysis for the Global Burden of Disease Study 2017. Lancet (London, England), 392(10159), 1789-1858. https://doi.org/10.1016/S0140-6736(18)32279-7

Giacaman, R. A., Muñoz-Sandoval, C., Neuhaus, K. W., Fontana, M., \& Chałas, R. (2018). Evidence-based strategies for the minimally invasive treatment of carious lesions: Review of the literature. Advances in Clinical and Experimental Medicine: Official Organ Wroclaw Medical University, 27(7), 1009-1016. https://doi.org/10.17219/ACEM/77022

Hayashi, M., Fujitani, M., Yamaki, C., \& Momoi, Y. (2011). Ways of enhancing pulp preservation by stepwise excavation--a systematic review. Journal of Dentistry, 39(2), 95-107. https://doi.org/10.1016/J.JDENT.2010.10.012

Hernández, M., Cobb, D., \& Jr, E. J. S. (2014). Current strategies in dentin remineralization. Journal of Esthetic and Restorative Dentistry : Official Publication of the American Academy of Esthetic Dentistry ... [et Al.], 26(2), 139-145. https://doi.org/10.1111/JERD.12095

Hoefler, V., Nagaoka, H., \& Miller, C. S. (2016). Long-term survival and vitality outcomes of permanent teeth following deep caries treatment with step-wise and partial-caries-removal: A Systematic Review. Journal of Dentistry, 54, 25-32. https://doi.org/10.1016/J.JDENT.2016.09.009

Ismail, A. I., Sohn, W., Tellez, M., Amaya, A., Sen, A., Hasson, H., \& Pitts, N. B. (2007). The International Caries Detection and Assessment System (ICDAS): an integrated system for measuring dental caries. Community Dentistry and Oral Epidemiology, 35(3), 170-178. https://doi.org/10.1111/J.16000528.2007.00347.X

Khokhar, M., \& Tewari, S. (2018). Outcomes of Partial and Complete Caries Excavation in Permanent Teeth: A 18 Month Clinical Study. Contemporary Clinical Dentistry, 9(3), 468-473. https://doi.org/10.4103/CCD.CCD_344_18

Kidd, E. (2000). The Cartwright Prize. Caries removal and the pulpo-dentinal complex. Dental Update, 27(10), 476-482. https://doi.org/10.12968/DENU.2000.27.10.476

Lee, H., Chalmers, N. I., Brow, A., Boynes, S., Monopoli, M., Doherty, M., Croom, O., \& Engineer, L. (2018). Person-centered care model in dentistry. BMC Oral Health, 18(1). https://doi.org/10.1186/S12903-018-0661-9

Loomans, B. A. C., Kreulen, C. M., Huijs-Visser, H. E. C. E., Sterenborg, B. A. M. M., Bronkhorst, E. M., Huysmans, M. C. D. N. J. M., \& Opdam, N. J. M. (2018). Clinical performance of full rehabilitations with direct composite in severe tooth wear patients: 3.5 Years results. Journal of Dentistry, 70, 97-103. https://doi.org/10.1016/J.JDENT.2018.01.001

Lygidakis, N. A. (2010). Treatment modalities in children with teeth affected by molar-incisor enamel hypomineralisation (MIH): A systematic review. European Archives of Paediatric Dentistry: Official Journal of the European Academy of Paediatric Dentistry, 11(2), 65-74. https://doi.org/10.1007/BF03262715

Machiulskiene, V., Campus, G., Carvalho, J. C., Dige, I., Ekstrand, K. R., Jablonski-Momeni, A., Maltz, M., Manton, D. J., Martignon, S., Martinez-Mier, E. A., Pitts, N. B., Schulte, A. G., Splieth, C. H., Tenuta, L. M. A., Ferreira Zandona, A., \& Nyvad, B. (2020). Terminology of Dental Caries and Dental Caries Management: Consensus Report of a Workshop Organized by ORCA and Cariology Research Group of IADR. Caries Research, 54(1), 7-14. https://doi.org/10.1159/000503309

Maltz, M., Garcia, R., Jardim, J. J., Paula, L. M. de., Yamaguti, P. M., Moura, M. S., Garcia, F., Nascimento, C., Oliveira, A., \& Mestrinho, H. D. (2012). Randomized trial of partial vs. stepwise caries removal: 3-year follow-up. Journal of Dental Research, 91(11), 1026-1031. https://doi.org/10.1177/0022034512460403

Maltz, M., Jardim, J. J., Mestrinho, H. D., Yamaguti, P. M., Podestá, K., Moura, M. S., \& Paula, L. M. de. (2013). Partial removal of carious dentine: a multicenter randomized controlled trial and 18-month follow-up results. Caries Research, 47(2), 103-109. https://doi.org/10.1159/000344013

Maltz, M., Koppe, B., Jardim, J. J., Alves, L. S., Paula, L. M. de., Yamaguti, P. M., Almeida, J. C. F., Moura, M. S., \& Mestrinho, H. D. (2018). Partial caries removal in deep caries lesions: a 5-year multicenter randomized controlled trial. Clinical Oral Investigations, 22(3), 1337-1343. https://doi.org/10.1007/S00784$017-2221-0$

Manton, D. (2013). Partial caries removal may have advantages but limited evidence on restoration survival. Evidence-Based Dentistry, 14(3), 74-75. https://doi.org/10.1038/SJ.EBD.6400948

Martignon, S., Bartlett, D., Manton, D. J., Martinez-Mier, E. angeles, Splieth, C., \& Avila, V. (2021). Epidemiology of Erosive Tooth Wear, Dental Fluorosis and Molar Incisor Hypomineralization in the American Continent. Caries Research, 55(1), 1-11. https://doi.org/10.1159/000512483

Mattos, J., Soares, G. M., \& Ribeiro, A. de A. (2014). Current status of conservative treatment of deep carious lesions. Dental Update, 41(5), 452-456. https://doi.org/10.12968/DENU.2014.41.5.452

Mendes, K. D. S., Silveira, R. C. de C. P., \& Galvão, C. M. (2008). Revisão integrativa: método de pesquisa para a incorporação de evidências na saúde e na enfermagem. Texto \& Contexto - Enfermagem, 17(4), 758-764. https://doi.org/10.1590/S0104-07072008000400018

Mesko, M. E., Cenci, M. S., Loomans, B., Opdam, N., \& Pereira-Cenci, T. (2016). Reabilitação oral do desgaste dentário severo com resina composta. Faculdade de Odontologia Da UPF, 21, 121-129. https://doi.org/doi.org/10.5335/rfo.v21i1.5023

Murdoch-Kinch, C. A., \& McLean, M. E. (2003). Minimally invasive dentistry. Journal of the American Dental Association (1939), 134(1), 87-95. https://doi.org/10.14219/JADA.ARCHIVE.2003.0021

Ngo, H., \& Opsahl-Vital, S. (2014). Minimal intervention dentistry II: part 7. Minimal intervention in cariology: the role of glass-ionomer cements in the preservation of tooth structures against caries. British Dental Journal, 216(10), 561-565. https://doi.org/10.1038/SJ.BDJ.2014.398 
Nyvad, B., Machiulskiene, V., \& Baelum, V. (1999). Reliability of a new caries diagnostic system differentiating between active and inactive caries lesions. Caries Research, 33(4), 252-260. https://doi.org/10.1159/000016526

Pozos-Guillén, A., Molina, G., Soviero, V., Arthur, R. A., Chavarria-Bolaños, D., \& Acevedo, A. M. (2021). Management of dental caries lesions in Latin American and Caribbean countries. Brazilian Oral Research, 35(suppl 01), 1-22. https://doi.org/10.1590/1807-3107BOR-2021.VOL35.0055

Ricketts, D., Lamont, T., Innes, N. P., Kidd, E., \& Clarkson, J. E. (2019). Operative caries management in adults and children. The Cochrane Database of Systematic Reviews, 7(7). https://doi.org/10.1002/14651858.CD003808.PUB4

Rindal, D. B., Gordan, V. V, Fellows, J. L., Spurlock, N. L., Bauer, M. R., Litaker, M. S., Gilbert, G. H., \& Group, D. C. (2012). Differences between reported and actual restored caries lesion depths: results from The Dental PBRN. Journal of Dentistry, 40(3), 248-254. https://doi.org/10.1016/J.JDENT.2011.12.015

Rosa, W. L. O. da, Lima, V. P., Moraes, R. R., Piva, E., \& Silva, A. F. da. (2019). Is a calcium hydroxide liner necessary in the treatment of deep caries lesions? A systematic review and meta-analysis. International Endodontic Journal, 52(5), 588-603. https://doi.org/10.1111/IEJ.13034

Sato, Y., \& Fusayama, T. (1976). Removal of dentin by fuchsin staining. Journal of Dental Research, 55(4), 678-683. https://doi.org/10.1177/00220345760550042301

Singh, S., Mittal, S., \& Tewari, S. (2019). Effect of Different Liners on Pulpal Outcome after Partial Caries Removal: A Preliminary 12 Months Randomised Controlled Trial. Caries Research, 53(5), 547-554. https://doi.org/10.1159/000499131

Slayton, R. L., Urquhart, O., Araujo, M. W. B., Fontana, M., Guzmán-Armstrong, S., Nascimento, M. M., Nový, B. B., Tinanoff, N., Weyant, R. J., Wolff, M. S., Young, D. A., Zero, D. T., Tampi, M. P., Pilcher, L., Banfield, L., \& Carrasco-Labra, A. (2018). Evidence-based clinical practice guideline on nonrestorative treatments for carious lesions: A report from the American Dental Association. Journal of the American Dental Association (1939), 149(10), 837-849.e19. https://doi.org/10.1016/J.ADAJ.2018.07.002

Souza, M. T. de., Silva, M. D. da., \& Carvalho, R. de. (2010). Integrative review: what is it? How to do it? Einstein (São Paulo), 8(1), 102-106. https://doi.org/10.1590/S1679-45082010RW1134

Tedesco, T. K., Reis, T. M., Mello-Moura, A. C. V., Silva, G. S. da, Scarpini, S., Floriano, I., Gimenez, T., Mendes, F. M., \& Raggio, D. P. (2020). Management of deep caries lesions with or without pulp involvement in primary teeth: a systematic review and network meta-analysis. Brazilian Oral Research, 35, 1-14. https://doi.org/10.1590/1807-3107BOR-2021.VOL35.0004

Tyas, M. J., Anusavice, K. J., Frencken, J. E., \& Mount, G. J. (2000). Minimal intervention dentistry--a review. FDI Commission Project 1-97. International Dental Journal, 50(1), 1-12. https://doi.org/10.1111/J.1875-595X.2000.TB00540.X

Veiga, N., Pereira, C., \& Amaral, O. (2015). Prevalence and Determinants of Dental Caries in Portuguese Children. Procedia - Social and Behavioral Sciences, 171, 995-1002. https://doi.org/10.1016/J.SBSPRO.2015.01.219

Villat, C., Attal, J.-P., Brulat, N., Decup, F., Doméjean, S., Dursun, E., Fron-Chabouis, H., Jacquot, B., Bolla, M. M., Plasse-Pradelle, N., Roche, L., MaucortBoulch, D., Nony, P., Gritsch, K., Millet, P., Gueyffier, F., \& Grosgogeat, B. (2016). One-step partial or complete caries removal and bonding with antibacterial or traditional self-etch adhesives: study protocol for a randomized controlled trial. Trials, 17(1). https://doi.org/10.1186/S13063-016-1484-0

Zhang, Q., Ou, Y.-J., \& Zhang, L. (2017). Curative effects of partial caries removal in permanent molars with deep dental caries. Shanghai Kou Qiang Yi Xue = Shanghai Journal of Stomatology, 26(3), 321-323. 